\title{
Lack of Drosophila cytoskeletal tropomyosin affects head morphogenesis and the accumulation of oskar mRNA required for germ cell formation
}

\section{Michael T.Tetzlaff', Herbert Jäckle and Michael J.Pankratz ${ }^{2}$}

\author{
Abteilung Molekulare Entwicklungsbiologie, Max-Planck-Institut für \\ biophysikalische Chemie, Postfach 2841, D-37018 Göttingen, \\ Germany \\ 'Present address: Baylor College of Medicine, One Baylor Plaza, \\ Houston, TX 77030, USA \\ ${ }^{2}$ Corresponding author
}

Drosophila encodes five muscle and one cytoskeletal isoform of the actin-binding protein tropomyosin. We have identified a lack-of-function mutation in the cytoskeletal isoform (cTmII). Zygotic mutant embryos show a defect in head morphogenesis, while embryos lacking maternal cTmII are defective in germ cell formation but otherwise give rise to viable adults. oskar mRNA, which is required for both germ cell formation and abdominal segmentation, fails to accumulate at the posterior pole in these embryos. nanos mRNA, however, which is required exclusively for abdominal segmentation, is localized at wild-type levels. These results indicate that head morphogenesis and the accumulation of high levels of oskar mRNA necessary for germ cell formation require tropomyosin-dependent cytoskeleton.

Keywords: actin cytoskeleton/germ cell formation/head morphogenesis/oskar mRNA localization/tropomyosin

\section{Introduction}

The cytoskeleton is critical for mediating all aspects of cell behavior, including cell division, cell movement and the transport of materials within cells (Bray, 1992; Stossel, 1993). In Drosophila, several mutations in genes encoding actin cytoskeletal components have been identified. They include spaghetti-squash, which encodes a regulatory light chain of non-muscle myosin necessary for cytokinesis (Karess et al., 1991), zipper, which encodes a non-muscle myosin heavy chain required for cell sheet movements during dorsal closure (Young et al., 1993) and chickadee, which encodes a profilin needed for transport of cell cytoplasm during oogenesis (Cooley et al., 1992). In the case of microtubule cytoskeletal components, their essential role has been shown for localizing mRNAs involved in germline determination and body patterning (reviewed in Macdonald, 1992; St Johnston, 1995). These two developmental processes are interconnected in Drosophila through the activity of the pole plasm, which is required for both germ cell formation and abdominal segmentation (reviewed in St Johnston, 1993). The assembly of the pole plasm is induced through localizing the mRNA of oskar (osk) (Lehmann and Nüsslein-Volhard, 1986) at the posterior pole of the developing oocyte (Ephrussi et al., 1991; Kim-Ha et al., 1991).

One component of the actin cytoskeleton is tropomyosin, an evolutionarily conserved protein that stabilizes filamentous actin structure and modulates its interaction with other actin-binding proteins (Bray, 1992). Tropomyosin gene disruptions in yeast result in the disappear-

A

\section{Organization of the Tropomyosin Locus}
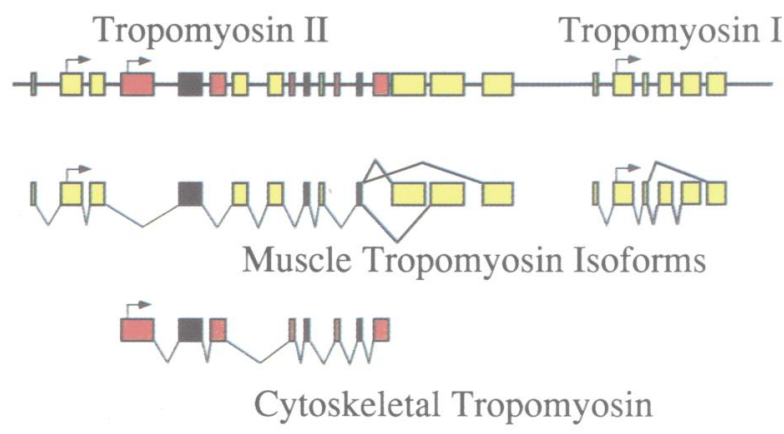

B

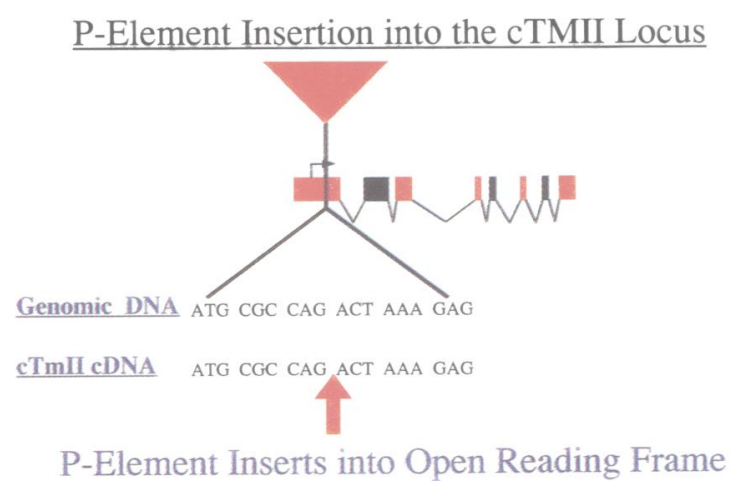

Fig. 1. The tropomyosin locus and the molecular lesion produced by the P-element. (A) The boxes represent exons and the arrows indicate the start of the coding region. Yellow boxes indicate exons unique to the muscle isoforms, the black boxes indicate exons shared by the muscle and the cytoskeletal isoforms and the red boxes indicate exons unique to the cytoskeletal isoform. The bent lines indicate splicing patterns. The tropomyosin II gene spans $\sim 27 \mathrm{~kb}$ (Hanke and Storti, 1988). (B) The $14 \mathrm{~kb}$ P-element enhancer trap construct [the red triangle; for details of the construct see Mlodzik and Hiromi (1992)] is inserted into the tropomyosin II gene at a position (red arrow) which would disrupt the cytoskeletal isoform $(c T m I I)$ open reading frame after the 20th amino acid. 


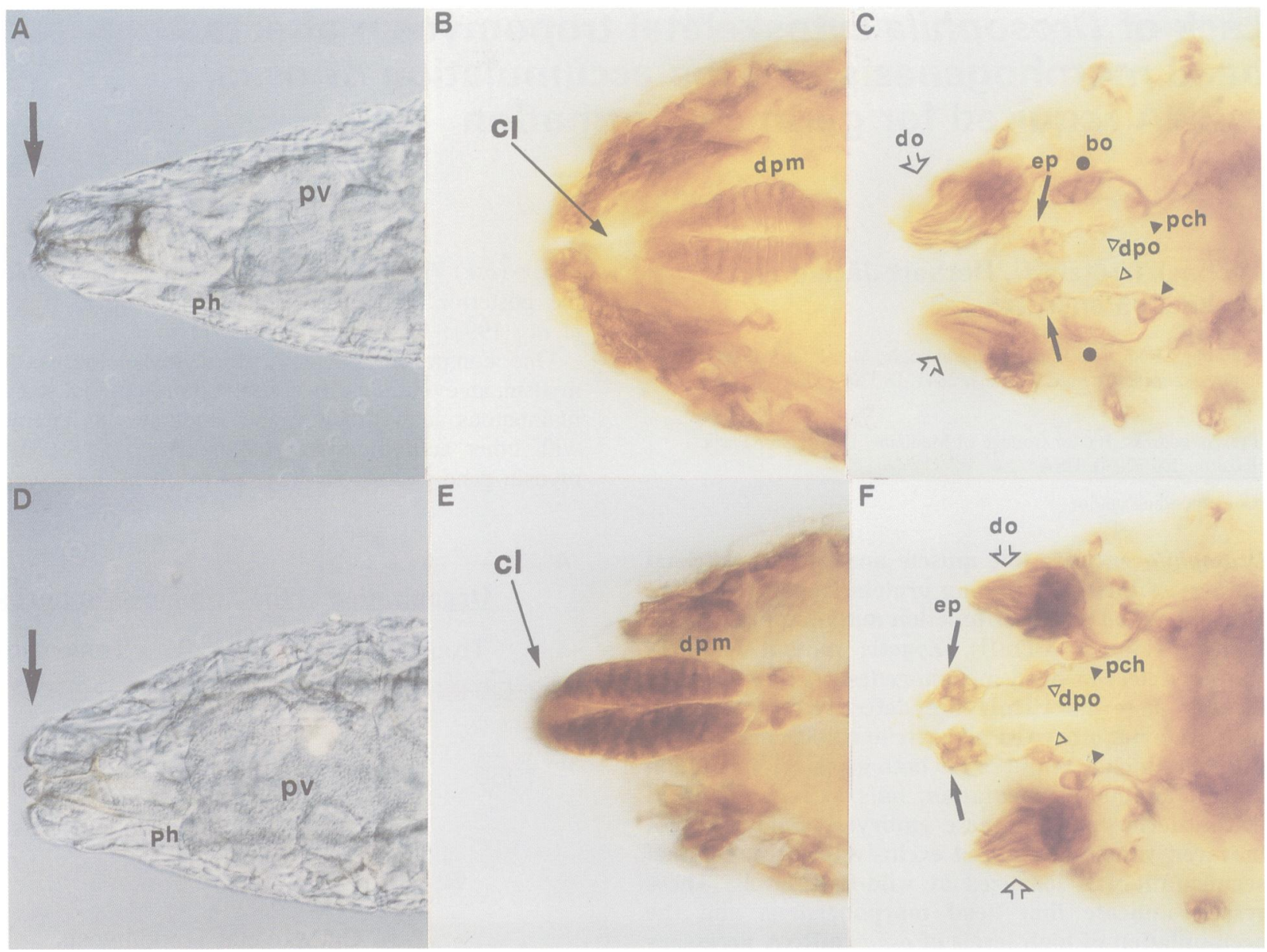

Fig. 2. Head morphogenesis phenotype of zygotic cTmII mutants. Wild-type (A) and mutant (D) first instar living larvae. The defect in the head (arrows) of mutant larva gives it a 'blockhead' appearance, rather than the normal pointed shape. Wild-type (B) and mutant (E) stage 17 embryos stained with muscle myosin heavy chain antibody. The clypeolabrum (cl) fails to be retracted into the body during head involution in the mutants, and the dorsal pharyngeal muscles (dpm) protrude out of the embryo. Wild-type $(\mathbf{C})$ and mutant $(\mathbf{F})$ stage 17 embryos stained with neuronal marker MAb22C10. In wild-type embryos the epiphysis (ep) comes to lie posterior to the dorsal organs (do), whereas in the mutants the relative positions of these organs are reversed. All of the major sense organs in the head, including the Bolwig organ (bo), dorsal pharyngeal organ (dpo) and the pharyngeal chordotonal organ (pch), are present in the mutants. The Bolwig organ is not visible in the mutants in a dorsal view since it comes to lie just below the dorsal organ. See text for details. Embryos are viewed dorsally, with anterior left. ph: pharynx; pv: proventriculus; cl: clypeolabrum; dpm: dorsal pharyngeal muscles; ep: epiphysis; bo: Bolwig organ; dpo: dorsal pharyngeal organ; pch: pharyngeal chordotonal organ.

ance of actin cables from the cytoskeleton and defects in cytokinesis (Liu and Bretscher, 1989; Balasubramanian et al., 1992). In mammalian cells various isoforms of tropomyosin associate with stress fibers and lamellipodia (Bray, 1992; Pittenger et al., 1994). Drosophila has five muscle isoforms and one non-muscle or cytoskeletal, isoform of tropomyosin (Karlik and Fyrberg, 1986; Hanke and Storti, 1988). The only mutation identified to date in Drosophila is in one of the muscle isoforms that affects the jump and indirect flight muscles (Karlik and Fyrberg, 1985; Tansey et al., 1987). Here we report the zygotic and maternal effects of a lack-of-function mutation in the cytoskeletal tropomyosin. Our results indicate that this actin-binding protein is required for specific cell movements during head morphogenesis and for accumulating high levels of osk mRNA at the posterior pole necessary for germ cell formation but not for abdominal development.

\section{Results}

\section{Identification of a cytoskeletal tropomyosin lack-of-function mutation}

In a search for genes which control the development and functioning of the larval feeding system (M.J.Pankratz, unpublished data), we isolated a lethal P-element line (Karpen and Spradling, 1992) in which the homozygous mutant larvae cannot feed due to the inability of the animals to suck food into the mouth atrium (see below). These mutants die as starving first instar larva. Mobilization of the P-element (Mlodzik and Hiromi, 1992) resulted in the reversion of the feeding defect, with the larvae developing into normal adults. This indicated that the insertion of the P-element is the cause of the mutant phenotype.

In order to clone the gene identified by the mutation, we isolated flanking sequences of the P-element by plasmid 


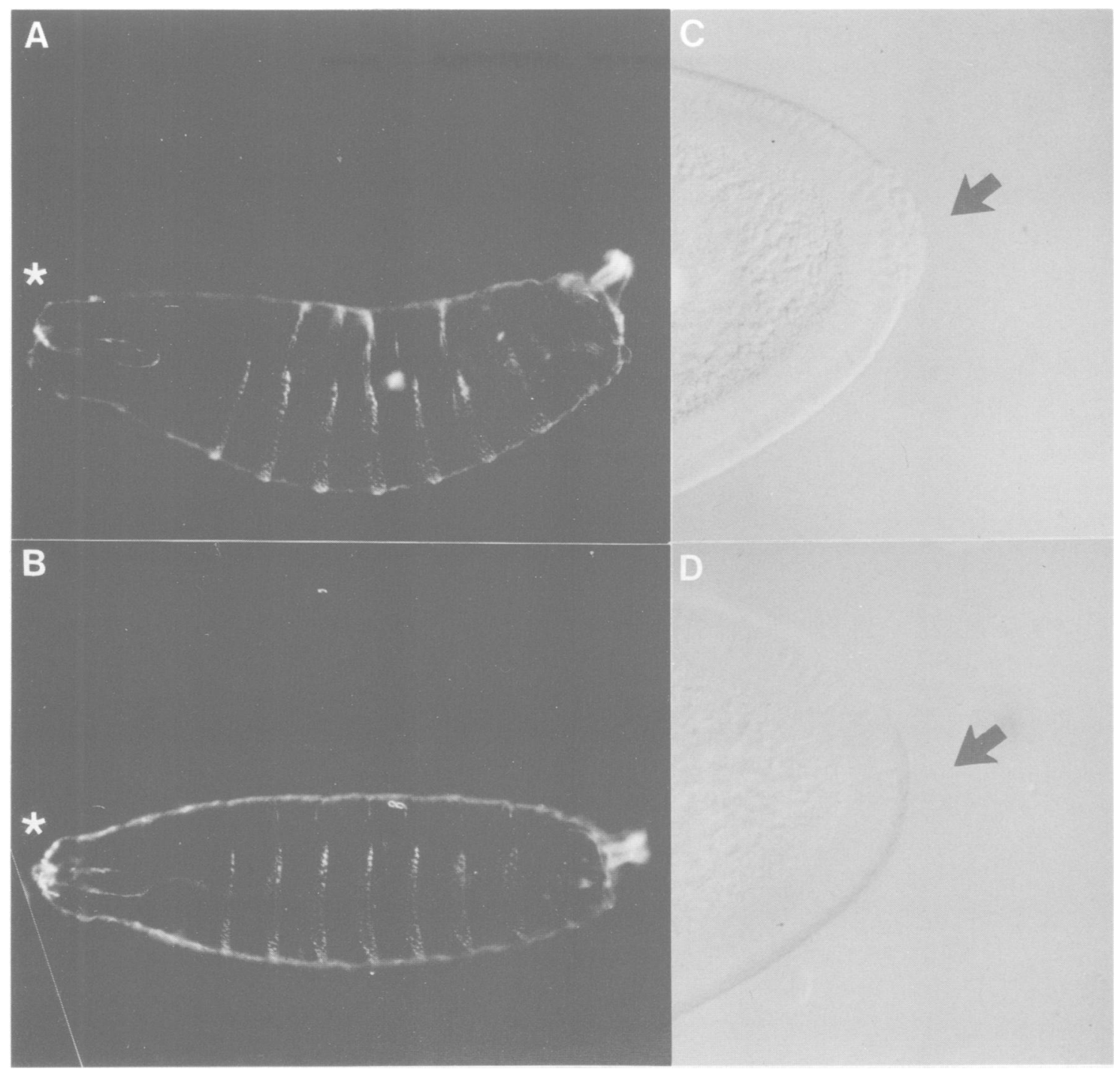

Fig. 3. Head and pole cell phenotypes of embryos lacking zygotic and maternal cTmII. (A) Cuticular phenotype of zygotic mutant. Note the characteristic head defect (asterisk), whereas the body segmentation pattern is normal. (B) Cuticular phenotype of mutant in which both maternal and zygotic components are eliminated. The head defect is the same as that of zygotic mutant alone, and the body segmentation pattern is also normal. (C) and (D) Posterior pole region of syncitial blastoderm in wild-type (C) and embryo lacking maternal cTmII (D). Note the pole cell defect (arrow) in the mutant. Both are dechorionated, devitellinized and mounted directly without histochemical staining.

rescue (Mlodzik and Hiromi, 1992). The subsequent sequencing of the plasmid rescued genomic DNA fragment indicated that the P-element has inserted into the tropomyosin locus (Figure 1A) at the cytogenetic interval $88 \mathrm{~F}$ at the right arm of the third chromosome. This locus consists of two neighboring genes termed tropomyosin I and II, each encoding various alternatively spliced isoforms of the actin-binding protein (Karlik and Fyrberg, 1986; Hanke and Storti, 1988). The tropomyosin II gene encodes three muscle isoforms through differential splicing of the same primary transcript. The only non-muscle, or cytoskeletal isoform encoded by the tropomyosin II gene $(c T m I I)$ derives from a different primary transcript under the control of a separate promoter (Hales et al., 1994). The P-element causing the mutant phenotype has inserted into an exon which is unique to $c$ TmII (Figure 1A). This insertion disrupts the single open reading frame after the 20th amino acid and thereby causes a non-muscle isoform- specific lack-of-function mutation, which we termed cTmIIP (Figure 1B).

\section{The lack of zygotic cytoskeletal tropomyosin results in a specific head defect}

$c T m I I$ is expressed nearly ubiquitously in a variety of tissues during development but with strong expression in the head region of late embryos (Hales et al., 1994; see below). Homozygous cTmIIP embryos develop into hatching larvae, but die during the first instar stage due to a defect in head morphogenesis (Figure 2). In wildtype embryonic development, the cells and tissues of the prospective head undergo a complex series of morphogenetic movements through which the entire head becomes tucked inside the body, a process called head involution (Jürgens and Hartenstein, 1993). The final step of this process occurs late in embryogenesis during stage 16 (Campos-Ortega and Hartenstein, 1985), when the pharynx 


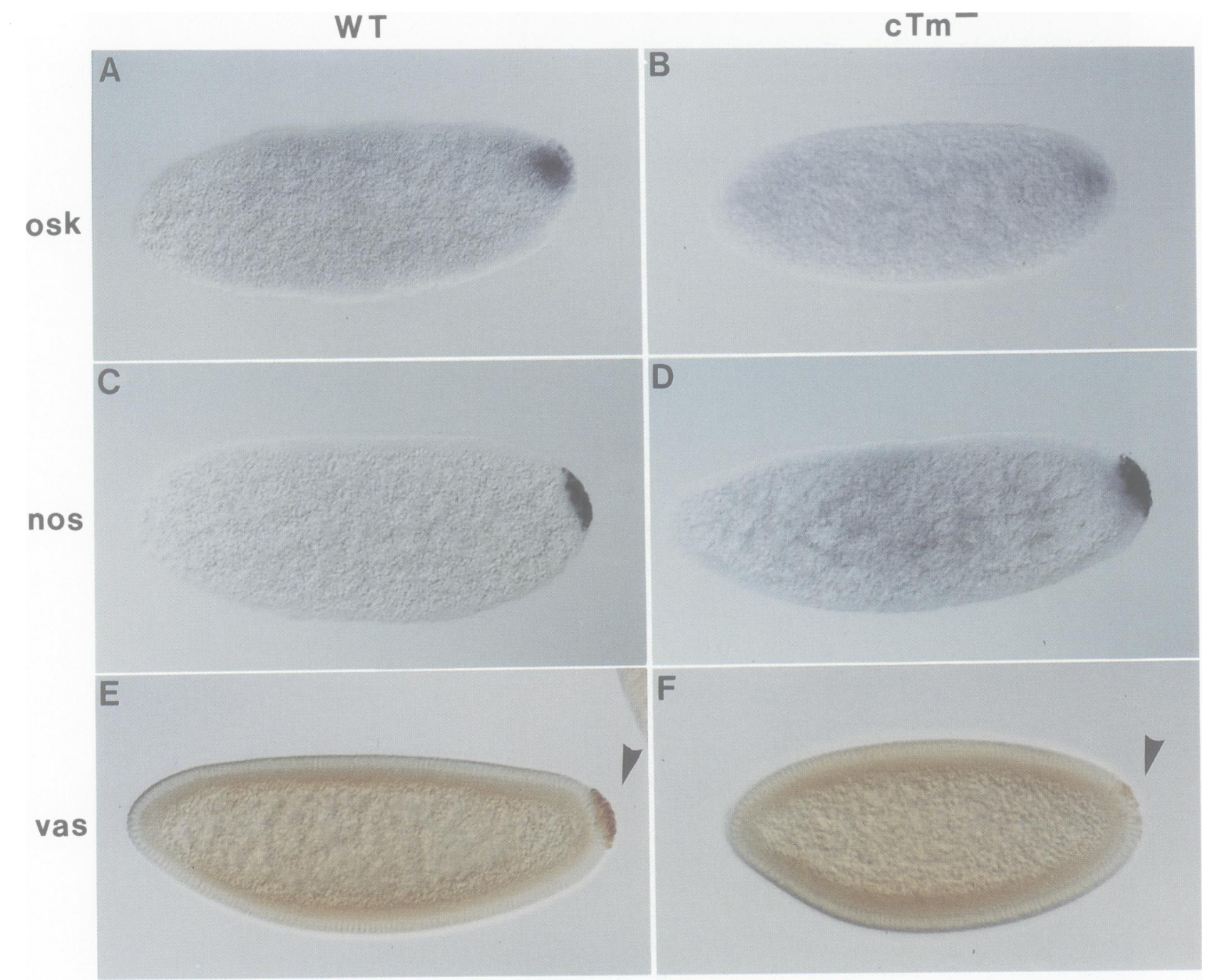

Fig. 4. Localization of osk, nos and vas gene products in embryos lacking maternal cTmII. In situ hybridization with osk (A and B) and nos (C and D) probes. Antibody stainings with anti-vas antibody (E and F). (A, C and E) are wild-type embryos. (B, D and F) are embryos lacking maternal cTmII. The staining of $o s k$ in the posterior pole of mutant embryos is greatly reduced compared with wild-type, whereas the nos staining is unaffected. In the absence of the pole cells, which normally take up nos mRNA, the mRNA becomes incorporated into the cytoplasm of the posterior terminal cells where it decays (data not shown). For vas, there is only a remnant staining in the posterior pole (arrowhead) of mutant embryo. Embryos in (A-D) are at the preblastoderm stage, and those in (E and F) are at syncitial and cellular blastoderm.

retracts into the main body. The phenotype of $c T m I I P$ embryos is indistinguishable from wild-type up to stage 16. However, the final step of retracting the pharynx into the body (Jürgens and Hartenstein, 1993) does not occur in the mutant embryos. This defect results in larvae with a protruding pharyngeal mass (Figure 2), which accounts for the inability of these animals to feed.

Morphological analysis with various cell markers indicated that all the identifiable parts of the head are present. Staining with muscle myosin heavy chain antibody (Kiehart and Feghali, 1986) revealed no gross structural defects in the muscle pattern (Figure $2 \mathrm{~B}$ and $\mathrm{E}$ ), and staining with the neuronal marker 22C10 (Zipursky et al., 1984) indicated that all the major sensory organs in the head (Schmidt-Ott et al., 1994) are present (Figure 2C and F). We did not detect any other gross morphogenetic defects in the embryo. The specificity of the mutation is further evidenced by the fact that the mutant embryos can hatch and the larva can move around normally and aggressively attempt to take in food (unpublished observation).

\section{The lack of maternal cytoskeletal tropomyosin affects germ cell formation}

cTmII is already expressed during oogenesis (Hales et. al., 1994; see below). Therefore, the late defect observed in zygotic $c$ TmIIP embryos could be due to the fact that the maternal component of cTmII provides sufficient activity to rescue the mutant embryos into the larval stage. Surprisingly, when we removed the maternal component of cTmII in addition to the zygotic one (Chou et al., 1993), no morphogenetic defects in addition to the one found in zygotic cTmIIP mutants were observed (Figure $3 \mathrm{~A}$ and $\mathrm{B})$. Indeed, when we removed only the maternal contribution, we obtained normal-looking adults. This indicates that the maternal component of $\mathrm{cTmII}$ has no lethal effect on embryonic, larval or adult development. However, embryos lacking maternal cTmII are defective 

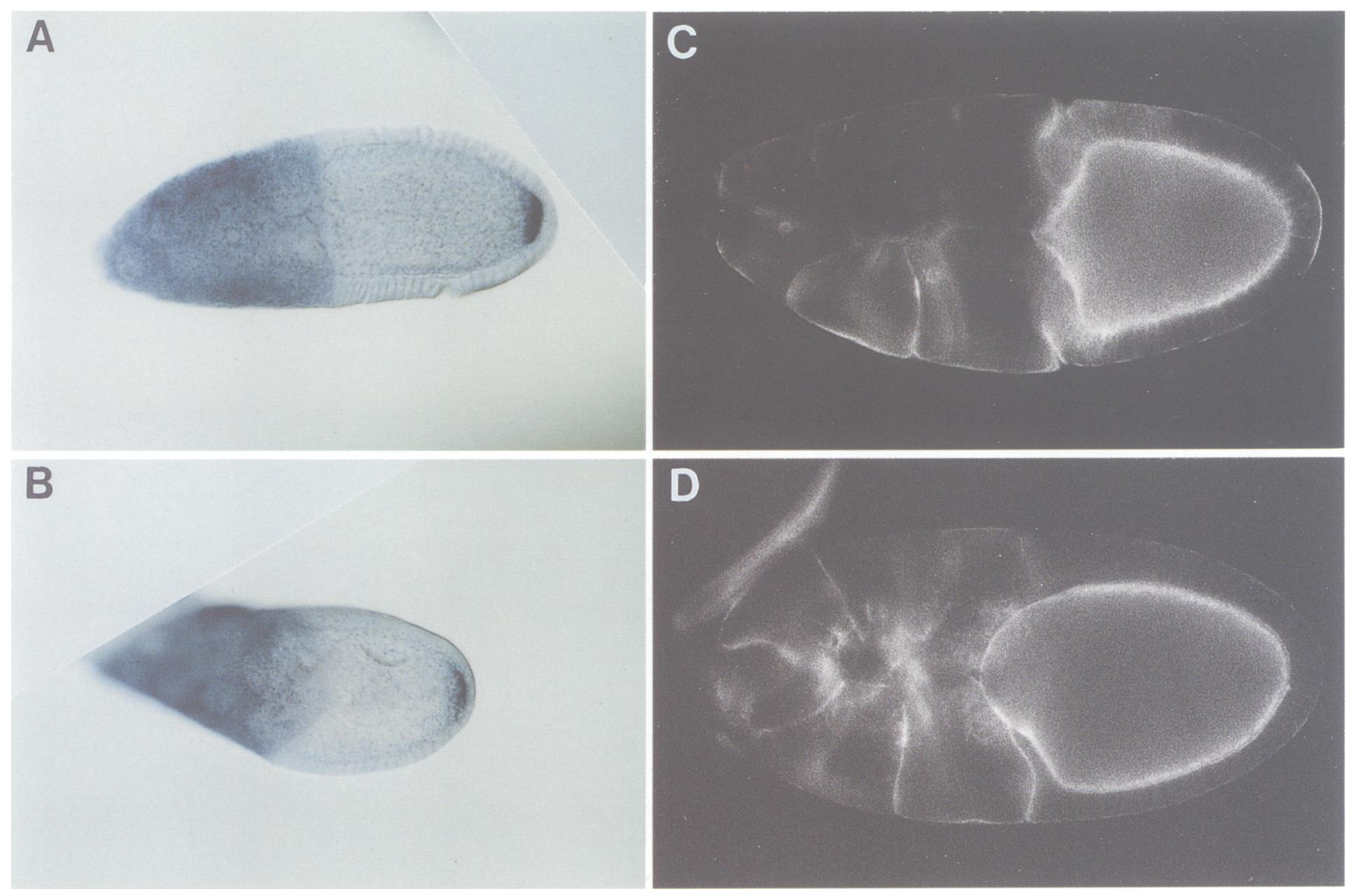

Fig. 5. In situ hybrization pattern of oskar mRNA and phalloidin staining of filamentous actin structure. (A) Wild-type ovary; note the localization of osk mRNA to the posterior pole. (B) $c T m I I$ mutant ovary showing osk mRNA staining at the posterior pole. (C) Wild-type ovary stained with rhodamine-phalloidin. (D) $c$ TmII mutant ovary stained with rhodamine-phalloidin; the staining pattern is essentially same as that of wild-type situation.

in the formation of the pole cells, which are the germline precursor cells. In wild-type development, pole cells are formed by pinching off from the rest of the cells at the posterior end of the embryo during blastoderm formation. As shown in Figure $3 \mathrm{C}$ and $\mathrm{D}$, pole cell formation is affected in embryos lacking maternal cTmII (see also Figure 4E and F).

\section{Maternal cTmll is required for oskar mRNA accumulation}

The formation of pole cells requires the activities of genes which are also necessary for abdominal segmentation (St Johnston and Nüsslein-Volhard, 1992; Pankratz and Jäckle, 1993; St Johnston, 1993). These genes comprise a cascade which is required to localize components of the pole plasm at the posterior pole of the embryo. A key component of the pole plasm is encoded by the gene osk (Lehmann and Nüsslein-Volhard, 1986), the mRNA of which is localized at the posterior pole and whose activity is critical for both pole cell formation and abdominal segmentation (Ephrussi et al., 1991; Kim-Ha et al., 1991; Ephrussi and Lehmann, 1992; Smith et al., 1992). A branchpoint in distinguishing the two developmental processes is the localization of mRNA for nanos (nos), a posterior determinant which is required only for abdominal segmentation and not germ cell formation (Lehmann and Nüsslein-Volhard, 1991; Wang and Lehmann, 1991). In embryos lacking maternal cTmII, the level of osk mRNA localized to the posterior pole is dramatically reduced as compared with wild-type embryos (Figure 4A and B). We also examined the expression of vasa (vas) protein, which is also required for both germ cell and abdominal development, and whose posterior localization depends on osk activity (Hay et al., 1988, 1990; Lasko and Ashburner, 1988 , 1990). Only remnant vas staining is seen in the posterior pole where pole cells would normally pinch out (Figure 4E and F).

The difference in the level of localized osk mRNA in wild-type and cTmII mutant condition is less dramatic in the oocytes than in the early embryos (Figure 5A and B), and the precise level of reduction is variable. Mislocalization of osk mRNA, as is found in gurken and cornichon mutant oocytes (Gonzalez-Reyes et al., 1995; Roth et al., 1995), was not observed. Furthermore, staining with phalloidin indicated no major alterations in filamentous actin structure (Figure 5C and D). Taken together, these results suggest that $\mathrm{cTmII}$ is required to anchor or maintain osk mRNA at the posterior pole, rather than in the actual transport from the anterior to the posterior pole. This view is supported by the expression pattern of the $c T m I I$ mRNA (Figure 6), which is localized at the posterior pole of the oocyte and early embryos.

\section{Discussion}

The head phenotype of zygotic cTmIIP mutants contrasts with the head defects associated with the various segmenta- 


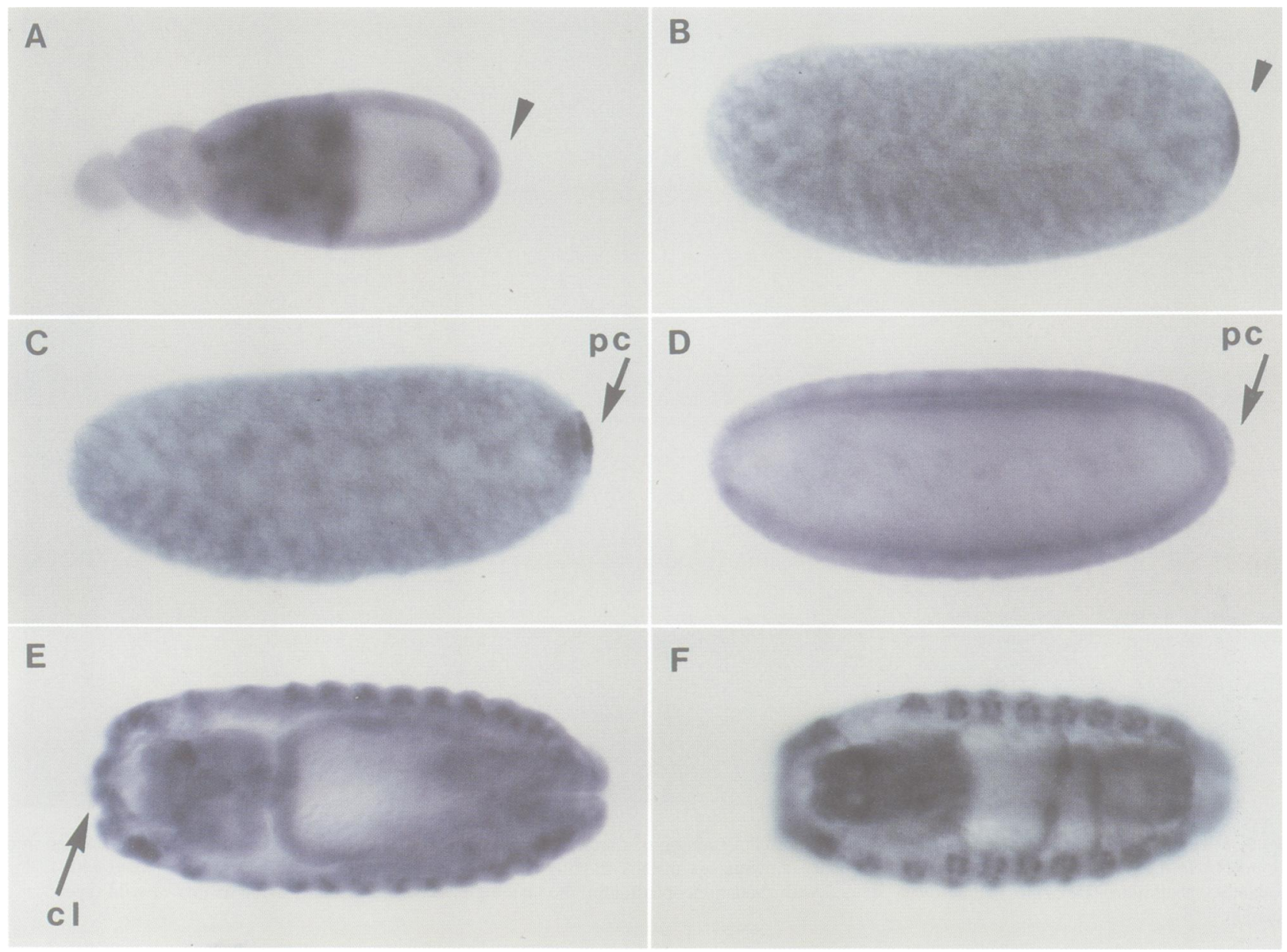

Fig. 6. In situ hybridization pattern of $c$ TmII mRNA in wild-type oocyte and embryos. (A) cTmII mRNA is localized to the posterior pole (arrowhead) of the oocyte. (B-D) cTmII mRNA expression pattern in early embryos. Note strong localization at the posterior pole (arrow) of freshly laid egg (B) and in the pole cells (C). The expression in the pole cells disappears at the beginning of gastrulation (D). (E-F) cTmII mRNA expression pattern in late embryos, showing staining in the clypeolabrum as well as in a variety of other tissues, including the midgut and brain. pc: pole cells, cl: clypeolabrum.

tion gene mutants (Jürgens and Hartenstein, 1993). In the latter case, the defects observed can be attributed to deletion of certain segmental regions of the head anlagen. In light of the known biochemical properties of the tropomyosins in actin binding and in regulating the cell contractile apparatus (Bray, 1992), the mutant phenotype of $c$ TmIIP embryos is most likely due to the failure of specific cells and tissues in the head to move per se, rather than as a secondary consequence arising from deletion of certain head structures. These observations indicate that the cytoskeletal tropomyosin functions in a specific aspect of cell movement during head involution.

The pole cell defect in embryos lacking the maternal cTmII is consistent with the view that the level of osk activity is reduced due to the strongly reduced level of osk mRNA accumulation at the posterior pole. On the other hand, the fact that these embryos do not have abdominal defects indicates that the drastic decrease in osk mRNA localization does not interfere with the abdominal segmentation process. It is known that the process of abdominal segmentation is less sensitive to defects in the posterior pole plasm than pole cell formation (Lehmann and Nüsslein-Volhard, 1986) and weak alleles of posterior group genes exist which are defective in germ cell formation but not abdominal segmentation (St Johnston, 1993).
Indeed, in embryos lacking maternal cTmII, nos mRNA is localized in a manner indistinguishable from that of the wild-type (Figure 4C and D). Our results show that the lack-of-function mutation in cytoskeletal tropomyosin causes a sufficient decrease in the level of osk mRNA accumulation to affect germ cell formation, but does not affect the localization of components specifically required for abdominal segmentation. Since such embryos give rise to viable adults, localization of components required for proper anterior segmentation and dorsal-ventral polarity (St Johnston and Nüsslein-Volhard, 1992; Roth et al., 1995; Gonzalez-Reyes et al., 1995) is also functionally unaffected. In view of the otherwise nearly ubiquitous expression of $c T m I I$, other possible functions may be hidden by functional redundancies between different components of the cytoskeleton.

At this point, the precise molecular mechanism underlying cTmII function is not known. cTmII could function at several steps in germ cell formation, including being part of a multi-protein complex that anchors osk mRNA (for reviews see Macdonald, 1992; Wilhelm and Vale, 1993; see also Webster et al., 1994). However, the function of the tropomyosin-dependent actin cytoskeleton appears to be different from that of the microtubule cytoskeleton in the intracellular localization of mRNAs 
required for germ cell formation and embryonic polarity. For example, it has been shown that the microtubuleassociated staufen protein (Ferrandon et al., 1994) is required for proper localization of osk mRNA as well as mRNA of bicoid, the key component of the maternal anterior segmentation system (St Johnston et al., 1989). Consistent with this, embryos from mutant staufen females have segment pattern defects in both the anterior and posterior regions (Schüpbach and Wieschaus, 1986; St Johnston et al., 1991). In contrast, the lack of cTmII activity affects germ cell formation but gives rise to viable adults. This suggests that the requirement for the tropomyosin-dependent cytoskeleton is restricted to those components generating the germ cells. Taken together, the findings to date suggest that the microtubule cytoskeleton plays the major role in the transport of materials to the poles (Pokrywka and Stephenson, 1991; Theurkauf et al., 1992; Clark et al., 1994; Ferrandon et al., 1994; Lane and Kalderon, 1994; Theurkauf, 1994; Gonzalez-Reyes et al., 1995; Roth et al., 1995; St Johnston, 1995), while the tropomyosin-dependent actin cytoskeleton is needed for properly anchoring or accumulating the transported mRNAs to ensure their high level required for germ cell formation. This finding is reminiscent of earlier studies in Xenopus (Yisraeli et al., 1990), showing the involvement of microtubules in translocating maternal $V g l \mathrm{mRNA}$ and of microfilaments in anchoring the mRNA.

\section{Materials and methods}

\section{Isolation and molecular analysis of the cTmIl mutant}

The cTmII mutant was isolated in a feeding screen where we assayed for mutants which cannot swallow properly (M.J.Pankratz, unpublished data). We used the dyed yeast feeding assay (Pankratz and Hoch, 1995) to screen the P-element lines, which were kindly provided by Allan Spradling. Chromosome localization of the rescued fragment hybridizes to the $88 \mathrm{~F}$ region in the right arm of the third chromosome (data not shown), which also corresponds to the tropomyosin locus. This P-line, 1(3)2299, is also lethal over the deficiency Df(3R)ea (obtained from K.Anderson) which uncovers this chromosomal region. Mobilization of the P-element was performed using standard genetic crosses by introducing a source of transposase (Mlodzik and Hiromi, 1992). The genomic region flanking the P-element insertion point was isolated by plasmid rescue (Mlodzik and Hiromi, 1992) and the rescued fragments of 1.6 and $3.6 \mathrm{~kb}$ from two independent rescue experiments were subcloned into pBST and sequenced. Both match the published sequence of cytoskeletal tropomyosin (Hanke and Storti, 1988) and indicate that the P-element had entered into the position encoding the 20th amino acid within the open reading frame (see Figure 1). Whole mount in situ hybridization of the rescued fragment in embryos (see Figure 6) showed the expression pattern of cytoskeletal tropomyosin cDNA as previously reported (Hales et al., 1994).

\section{Immunocytochemistry}

The antibody stainings were done as previously described (Macdonald and Struhl, 1986). The in situ hybridization was carried out according to the method of Tautz and Pfeifle (1989), with minor modification for oocytes (Suter and Steward, 1991). DNA probes were used for detecting osk and nos mRNAs, while RNA probe was used for detecting $c T m$ mRNAs. For the latter the probe was derived from a $1.6 \mathrm{~kb}$ plasmid rescued genomic fragment that contains the $c T m$-specific first exon. The rhodamine-phalloidin staining was performed as previously described (Verheyen and Cooley, 1994). The osk and nos probes were gifts of R.Lehmann, vas antibody was a gift of P.Lasko, muscle myosin heavy chain antibody was a gift of D.Kiehart and 22C10 a gift of N.Patel.

\section{Germline clones}

Embryos lacking maternal cTmII were generated by the DFS-FLP method of Chou et al. (1993) using the original P-element line recombined onto the FRT82B chromosome, and the $\mathrm{P}$ (ovoD1) insertion on the FRT82B chromosome. A single heat shock was delivered at $37^{\circ} \mathrm{C}$ for $2 \mathrm{~h}$ during second and third larval instar stages. Embryos lacking both maternal and zygotic contribution were generated by fertilizing mutant germline clones with 1(3)2299/TM3Sb males, while those lacking only the maternal contribution were generated by fertilization with wildtypes males.

\section{Acknowledgements}

M.J.P. is very grateful to Allan Spradling for allowing him to screen through the collection of the P-element lines, R.Lehmann, P.Lasko, D.Kiehart, N.Patel and R.Storti for reagents and probes, K.Anderson for $e a$ deficiency stock, G.Dowe for sequencing, I.Gajewsky for photography, and M.Affolter, C.Hartmann, U.Häcker, S.Fellert and M.Hoch for their help during the course of this work. We are also very grateful to N.Perrimon for the stocks that allowed us to generate the germline clones. We especially thank Anne Ephrussi and Miklos Erdelyi for the open exchange of information concerning the role of TmII in oskar mRNA localization. M.T.T. wishes to thank Dr J.Lupski, Dr C.T.Caskey and the MD-PhD Program at Baylor College of Medicine for allowing time for this work to be completed and to D.Schmucker, M.Hoch and R.Rivera-Pomar for their contributions; a special thanks to Dr J.K.Skipper for support and encouragement. This work was supported by Max Planck Gesellschaft.

\section{References}

Balasubramanian,M., Helfman,D. and Hemmingsen,S. (1992) A new tropomyosin essential for cytokinesis in the fission yeast $S$. pombe. Nature, 360, 84-87.

Bray,D. (1992). Cell Movements. Garland Publishing Inc., New York. Campos-Ortega,J. and Hartenstein,V. (1985) The Embryonic Development of Drosophila melanogaster. Springer-Verlag, Berlin.

Chou,T.-B., Noll,E. and Perrimon,N. (1993) Autosomal P(ovoD1) dominant female-sterile insertions in Drosophila and their use in generating germ-line chimeras. Development, 119, 1359-1369.

Clark,I., Giniger,E., Ruohola-Baker,H., Jan,L. and Jan,Y. (1994) Transient posterior localisation of a kinesin fusion protein reflects anteroposterior polarity of the Drosophila oocyte. Curr. Biol., 4, 289-300.

Cooley,L., Verheyen,E. and Ayers,K. (1992) chickadee encodes a profilin required for intercellular cytoplasm transport during Drosophila oogenesis. Cell, 69, 173-184.

Ephrussi,A. and Lehmann,R. (1992) Induction of germ cell formation by oskar. Nature, 358, 387-392.

Ephrussi,A., Dickinson,L. and Lehmann,R. (1991) oskar organizes the germ plasm and directs localization of the posterior determinant nanos. Cell, 66, 37-50.

Ferrandon,D., Elphick,L., Nüsslein-Volhard,C. and St Johnston,D. (1994) Staufen protein associates with the 3'UTR of bicoid mRNA to form particles that move in a microtubule-dependent manner. Cell, 79, 1221-1232.

Gonzalez-Reyes,A., Elliott,H., St Johnston,D. (1995) Polarization of major body axes in Drosophila by gurken-torpedo signalling. Nature, 375, 654-658.

Hales,K., Meredith,J. and Storti,R. (1994) Transcriptional and posttranscriptional regulation of maternal and zygotic cytoskeletal tropomyosin mRNA during Drosophila development correlates with specific morphogenic events. Dev. Biol., 165, 639-653.

Hanke,P. and Storti,R. (1988) The Drosophila melanogaster tropomyosin II gene produces multiple proteins by use of alternative tissue-specific promoters and alternative splicing. Mol. Cell. Biol., 8, 3591-3602.

Hay,B., Jan,L. and Jan,J. (1988) sequence similarity to ATP-dependent helicases. Cell, 55, 577-587.

Hay,B., Jan,L. and Jan,Y. (1990) Localization of vasa, a component of Drosophila polar granules, in maternal-effect mutants that alter embryonic anteroposterior polarity. Development, 109, 425-433.

Jürgens,G. and Hartenstein,V. (1993) The terminal regions of the body pattern. In Bate,M. and Martinez-Arias,A. (eds), The Development of Drosophila melanogaster. Cold Spring Harbor Laboratory Press, Cold Spring Harbor, NY, pp. 687-746.

Karlik,C. and Fyrberg,E. (1985) An insertion within a variably spliced Drosophila tropomyosin gene blocks accumulation of only one encoded isoform. Cell, 41, 57-66.

Karlik,C. and Fyrberg,E. (1986) Two Drosophila melanogaster 
tropomyosin genes: structural and functional aspects. Mol. Cell. Biol., 6, 1965-1973.

Karpen,G. and Spradling,A. (1992) Analysis of subtelomeric heterochromatin in the Drosophila minichromosome Dp1 187 by single $P$ element insertional mutagenesis. Genetics, 132, 737-753.

Karess,R., Chang,X., Edwards,K., Kulkarni,S., Aguilera,I. and Kiehart,D. (1991) The regulatory light chain of non-muscle myosin is encoded by spaghetti-squash, a gene require for cytokinesis in Drosophila. Cell, 65, 1177-1189.

Kiehart,D. and Feghali,R. (1986) Cytoplasmic myosin from Drosophila melanogaster. J. Cell. Biol., 103, 1517-1525.

Kim-Ha,J., Smith,J. and Macdonald,P. (1991) oskar mRNA is localized to the posterior pole of the Drosophila oocyte. Cell, 66, 23-35.

Lane,M.E. and Kalderon,D. (1994) RNA localization along the anteroposterior axis of the Drosophila oocyte requires PKA-mediated signal transduction to direct normal microtubule organization. Genes Dev., 8, 2986-2995.

Lasko,P. and Ashburner,M. (1988) The product of the Drosophila gene vasa is very similar to eukaryotic initiation factor-4A. Nature, 335, 611-617.

Lasko,P. and Ashburner,M. (1990) Posterior localization of vasa protein correlates with, but is not sufficient for, pole cell development. Genes Dev., 4, 905-921.

Lehmann,R. and Nüsslein-Volhard,C. (1986) Abdominal segmentation, pole cell formation, and embryonic polarity require the localized activity of oskar, a maternal gene in Drosophila. Cell, 47, 141-152.

Lehmann,R. and Nüsslein-Volhard,C. (1991) The maternal gene nanos has a central role in posterior pattern formation in the Drosophila embryo. Development, 112, 679-691.

Liu,H. and Bretscher,A. (1989) Disruption of the single tropomyosin gene in yeast results in the disappearance of actin cables from the cytoskeleton. Cell, 57, 233-242.

Macdonald,P. (1992) The means to the ends: localization of maternal messenger RNAs. Semin. Dev. Biol., 3, 413-424.

Macdonald,P. and Struhl,G. (1986) A molecular gradient in early Drosophila embryos and its role in specifying body pattern. Nature, 324, 537-545.

Mlodzik,M. and Hiromi,Y. (1992) Enhancer trap method in Drosophila: Its application to neurobiology. Methods Neurosci., 9, 397-413.

Pankratz,M.J. and Hoch,M. (1995) Control of epithelial morphogenesis by cell signaling and integrin molecules in the Drosophila foregut. Development, 121, 1885-1898.

Pankratz,M.J. and Jäckle,H. (1993) Blastoderm segmentation. In Bate,M. and Martinez-Arias,A. (eds), The Development of Drosophila melanogaster. Cold Spring Harbor Laboratory Press, Cold Sping Harbor, NY, pp. 467-516.

Pittenger,M., Kazzaz,J. and Helfman,D. (1994) Functional properties of non-muscle tropomyosin isoforms. Curr. Opin. Cell. Biol., 6, 96-104.

Pokrywka,N. and Stephenson,E. (1991) Microtubules mediate the localization of bicoid RNA during Drosophila oogenesis. Development, $113,55-66$.

Roth,S., Neuman-Silberberg,F., Barcelo,G. and Schüpbach,T. (1995) cornichon and the EGF receptor signaling process are necessary for both anterior-posterior and dorsal-ventral pattern formation in Drosophila. Cell, 81, 967-978.

Schmidt-Ott,U., Gonzalez-Gaitan,M., Jäckle,H. and Technau,G. (1994) Number, identity and sequence of the Drosophila head segments. Proc. Natl Acad. Sci. USA, 91, 8363-8367.

Schüpbach,T. and Wieschaus,E. (1986) Maternal effect mutations altering the anteriorposterior pattern of the Drosophila embryo. Roux's Arch. Dev. Biol., 195, 302-317.

Smith,J., Wilson,J. and Macdonald,P. (1992) Overexpression of oskar directs ectopic activation of nanos and presumptive pole cell formation in Drosophila embryos. Cell, 70, 849-859.

St Johnston,D. (1993) Pole plasm and the posterior group genes. Bate,M. and Martinez-Arias,A. (eds), In The Development of Drosophila melanogaster. Cold Spring Harbor Laboratory Press, Cold Spring Harbour, NY, pp. 325-364.

St Johnston,D. (1995) The intracellular localization of messenger RNAs. Cell, 81, 161-170.

St Johnston,D. and Nüsslein-Volhard,C. (1992) The origin of pattern and polarity in the Drosophila embryo. Cell, 68, 201-219.

St Johnston,D., Driever,W., Berleth,T., Richstein,S. and NüssleinVolhard,C. (1989) Multiple steps in the localization of bicoid RNA to the anterior pole of the Drosophila oocyte. Development, Suppl., 107, 13-19.

St Johnston,D., Beuchle,D. and Nüsslein-Volhard,C. (1991) Staufen, a gene required to localize maternal RNAs in the Drosophila egg. Cell, 66, 51-63.

Stossel,T. (1993) On the crawling of animal cells. Science, 260, 10861094.

Suter,B. and Steward,R. (1991) Requirement for phosphorylation and localization of the Bicaudal-D protein in Drosophila oocyte differentiation. Cell, 67, 917-926.

Tansey,T., Mikus,M., Dumoulin,M. and Storti,R. (1987) Transformation and rescue of a flightless Drosophila tropomyosin mutant. EMBO J., 6, 1375-1385.

Tautz,D. and Pfeifle,C. (1989) A non-radioactive in situ hybridization method for the localization of specific RNAs reveals translational control of the segmentation gene hunchback. Chromosoma, 98, 81-85.

Theurkauf,W. (1994) Premature microtubule-dependent cytoplasmic streaming in cappuccino and spire mutant oocytes. Science, 265, 2093-2096.

Theurkauf,W., Smiley,S., Wong,M. and Alberts,B. (1992) Reorganization of the cytoskeleton during Drosophila oogenesis: implications for axis specification and intercellular transport. Development, 115, 923-936.

Verheyen,E. and Cooley,L. (1994) Looking at oogenesis. In Goldstein,L. and Fyrberg,E. (eds), Drosophila melanogaster: Practical Uses in Cell and Molecular Biology. Academic Press, San Diego, pp. 545-561.

Wang,C. and Lehmann,R. (1991) Nanos is the localized posterior determinant in Drosophila. Cell, 66, 637-647.

Webster,P., Suen,J. and Macdonald,P. (1994) Drosophila virilis oskar transgenes direct body patterning but not pole cell formation or maintenance of mRNA localization in D. melanogaster. Development, 120, 2027-2037.

Wilhelm,J. and Vale,R. (1993) RNA on the move: the mRNA localization pathway. J. Cell Biol., 123, 269-274.

Yisraeli,J., Sokol,S. and Melton,D. (1990) A two-step model for the localization of maternal mRNA in Xenopus oocytes: involvement of microtubules and microfilaments in the translocation and anchoring of $\mathrm{Vg} 1$ mRNA. Development, 108, 289-298.

Young,P., Richman,A., Ketchum,A. and Kiehart,D. (1993) Morphogenesis in Drosophila requires nonmuscle myosin heavy chain function. Genes Dev., 7, 29-41.

Zipursky,S., Venkatesh,T., Teplow,D. and Benzer,S. (1984) Neuronal development in the Drosophila retina: monoclonal antibodies as molecular probes. Cell, 36, 15-26.

Received on September 27, 1995; revised on November 21, 1995 